\title{
HACIA UNA APROXIMACIÓN DE LAS PRIMERAS ETAPAS DE REDUCCIÓN LÍTICA EN EL CAÑADÓN DE LA MINA, LOCALIDAD ARQUEOLÓGICA LA MARÍA, MESETA CENTRAL DE SANTA CRUZ, ARGENTINA.
}

\author{
ARIEL D. FRANK*, FABIANA SKARBUN" Y MATÍAS F. PAUNERO
}

\begin{abstract}
RESUMEN
En este trabajo se analiza la funcionalidad de la Cantera de Sílex del Sector Cañadón de la Mina, Localidad Arqueológica La María, Meseta Central de Santa Cruz, Argentina. Se examinan las características tecnológicas de los restos presentes allí y se evalúa si la cantera funcionó sólo como fuente de aprovisionamiento o también como taller lítico de los estadios iniciales en el proceso de elaboración de instrumentos. Además, se explora su relación con el sitio Casa del Minero 1, ubicado en el mismo sector. De manera más general, se busca aportar datos que permitan analizar a largo plazo las estrategias de aprovisionamiento y reducción de las materias primas, las estrategias de uso de espacios específicos como canteras y la planificación de ciertas actividades relacionadas a la organización de la tecnología lítica de los grupos que habitaron esta región.
\end{abstract}

PALABRAS CLAVE: Tecnología lítica, Cantera, Disponibilidad, Materias Primas.

TOWARDS AN APPROXIMATION OF THE FIRST STAGES OF THE LITHIC REDUCTION AT CAÑADÓN DE LA MINA, LA MARÍA ARCHAEOLOGICAL LOCALITY, SANTA CRUZ CENTRAL PLATEAU, ARGENTINA.

\begin{abstract}
This paper studies the functionality of a flint quarry (Cantera de Sílex) from Cañadón de la Mina Sector, La María Archaeological Locality, Santa Cruz Central Plateau, Argentina. The technological characteristics of the remains found there are analyzed. It is examined if the quarry worked only as a source of raw material or if it was used also as a lithic workshop for the first stages in the toolmaking process. The relationship with Casa del Minero 1 site, located at the same sector, is also examined. More generally, this paper intends to add valuable data which will allow to evaluate in the long term the raw material sourcing and the reduction strategies, as well as the strategies for the use of specific spaces such as quarries and the planning of certain activities related to the organization of the lithic technology of the groups that inhabited this region.
\end{abstract}

KEY WORDS: Lithic Technology, Quarry, Availability, Raw Materials.

Departamento Científico de Arqueología. Facultad de Ciencias Naturales y Museo. UNLP. Paseo del Bosque s/n. 1900. La Plata. Argentina. Email: arieldavidfrank@yahoo.com.ar; fskarbun@intepla.com; mat_tito@yahoo.com.ar 


\section{INTRODUCCIÓN}

Este trabajo se encuadra dentro del proyecto "Investigaciones Arqueológicas en la Meseta Central de Santa Cruz: Pasado Humano y Comunicación" dirigido por R. S. Paunero, que tiene como fines comprender cómo fueron los procesos de ocupación humana de los distintos ambientes a lo largo del tiempo, cuáles fueron las distintas estrategias de movilidad, subsistencia y uso del espacio implementadas, cómo fueron los patrones de asentamiento, qué expresiones artísticas y simbólicas realizaron estos grupos y cuál fue la organización tecnológica de los grupos cazadores recolectores que habitaron la región.

Las investigaciones llevadas a cabo por este equipo se han realizado en los últimos años principalmente en la localidad arqueológica La María, la cual ha sido subdividida en diversos sectores desde el punto de vista ambiental, paisajístico y arqueológico (Paunero et al. 2005). Esta localidad se caracteriza por su riqueza y alta visibilidad arqueológica: gran cantidad de pinturas rupestres en cuevas y aleros, alta densidad de artefactos líticos en superficie, pircas y restos de ocupaciones de principios del siglo XX. Uno de los rasgos más salientes es la presencia de

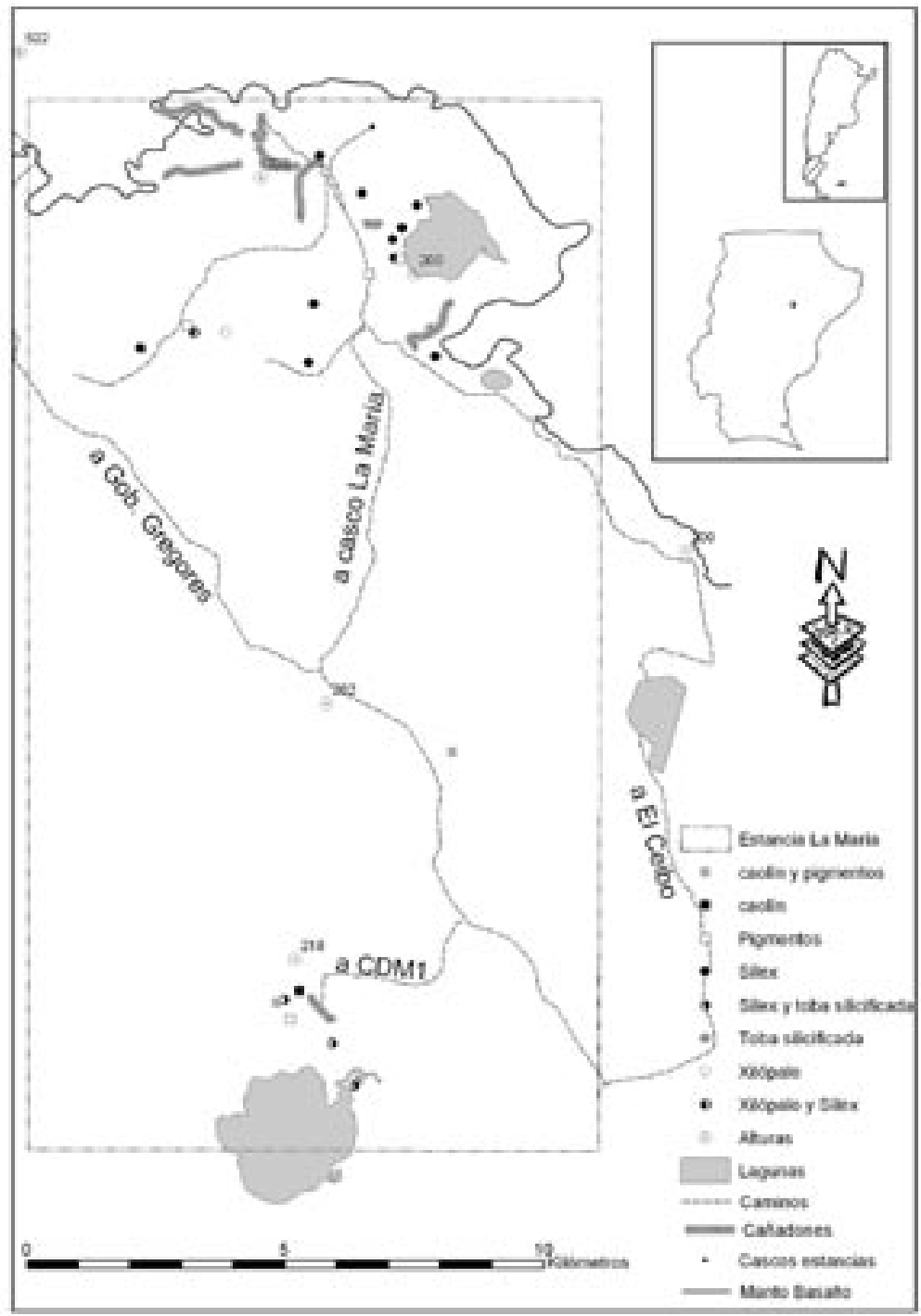

Fig. 1. Mapa de Canteras de la Localidad Arqueológica La María. 
abundantes afloramientos de materias primas líticas que presentan claros signos de aprovechamiento, por lo cual se infiere que muy probablemente hayan sido usadas como canteras líticas en el pasado (ver Fig. 1). En general, son afloramientos de materias primas silíceas (sílex, tobas silicificadas y xilópalos) de buena calidad para la talla.

El estudio de las fuentes de aprovisionamiento se ha vuelto un aspecto importante de la arqueología patagónica en general y de Santa Cruz en particular. Es así como se pueden observar publicaciones acerca del reconocimiento de fuentes de aprovisionamiento de variadas materias primas (obsidiana, basalto, dacita, andesita, xilópalo, entre otras). Estos estudios han utilizado diversos métodos como análisis geoquímicos (Espinosa y Goñi 1999, Franco y Aragón 2004, Stern 2004), cortes delgados (Belardi y Carballo Marina 2005) mapas geológicos (Cattáneo 2004) o de terreno mediante prospecciones o transectas (Espinosa y Goñi 1999; Espinosa et al. 2001). Fueron realizados trabajos con un enfoque regional, buscando identificar grandes áreas donde se presenta un tipo de roca determinado, delimitando, así, zonas de potencial provisión de estos recursos. También se ha llevado a cabo el estudio puntual de canteras específicas, analizando las características tecnológicas de los restos allí localizados (Espinosa et al. 2001, Hermo 2005). Ambos enfoques son complementarios y juntos colaboran para tener una mejor comprensión del modo en que los antiguos pobladores se aprovisionaron de los recursos líticos. Es necesario, por lo tanto, profundizar en el estudio tecnológico de las canteras como parte de una estrategia global de análisis de las fuentes de aprovisionamiento líticas de una región.

En este artículo se busca evaluar las hipótesis propuestas en trabajos anteriores acerca de la funcionalidad de la Cantera de Sílex del Sector Cañadón de la Mina, así como examinar cuál fue su relación con el sitio Casa del Minero 1 (CDM1). A partir de observaciones efectuadas en la cantera y de la excavación de un sector de $4 \mathrm{~m}^{2}$, se sugirió que esta cantera ha servido no sólo como sitio de extracción sino también como taller lítico de los estadios iniciales en los procesos de elaboración de instrumentos (Paunero et al. 2004). Además se ha propuesto que la materia prima proveniente de esta cantera fue intensamente utilizada en los procesos de producción lítica desde las ocupaciones tempranas del sitio CDM1.
De manera más general, el objetivo a largo plazo es analizar las estrategias de aprovisionamiento y reducción de las materias primas, así como las referidas al uso de espacios específicos como canteras o talleres y a la planificación de las actividades relacionadas con la accesibilidad a los recursos y a la movilidad, dentro de la organización de la tecnología lítica (Kelly 1988; Nelson 1991) de los grupos que habitaron el área desde las primeras ocupaciones.

Los grupos humanos implementan distintas estrategias para organizar su tecnología lítica. En este sentido, Nelson (1991) propone que existen dos estrategias principales dentro de un continuum: conservación y expeditividad. Entre las variables que influyen sobre estas estrategias se consideran el patrón de asentamiento (Binford 1977, 1980), la movilidad de los grupos, la función de los instrumentos (Kelly 1988) y la disponibilidad, accesibilidad, cantidad y calidad de las materias primas en el ambiente (Bamforth 1986; Kelly 1988; Andrefsky 1991, 1994). De esta manera, estas características de las materias primas son importantes en las estrategias de aprovisionamiento, así como en los procesos de producción, uso y descarte y en la morfología final de los instrumentos. También se ha propuesto la existencia de comportamientos oportunísticos, como una respuesta tecnológica no planeada ante una necesidad inmediata no anticipada ni prevista, mientras que la tecnología expeditiva sería una respuesta planeada a una tarea esperada (Nelson 1991). Sin embargo se ha planteado (Bousman 1993; Nelson 1991) que es difícil proponer un criterio independiente que separe estas dos estrategias basado en las condiciones morfológicas de los artefactos. Si son usadas estrategias tecnológicas expeditivas, entonces es necesario que exista materia prima disponible (Bousman 1993).

Desde este punto de vista, Andrefsky (1994) considera que en áreas que presentan abundante materia prima de buena calidad, la mayor parte de los artefactos, sin importar su función, serían realizados con dicha materia prima. En estos ambientes es esperable que se hayan utilizado estrategias más expeditivas. La Meseta Central y particularmente el área de estudio, pueden ser incluidas dentro de este tipo de ambientes (Cardich y Paunero 2000). Esto no impide que se hayan implementado estrategias de formatización con mayor inversión de energía 
en relación a prácticas de subsistencia particulares o para la producción de instrumentos con una función específica. Por lo tanto, en las primeras etapas de producción, expresadas en las canteras, se deberían encontrar principalmente núcleos poliédricos no agotados, con diferentes estadios de reducción; productos de talla no estandarizados, poca bifacialidad, muchos desechos e instrumentos poco formatizados. De cualquier manera, el modo de explotación de las canteras dependerá tanto de las características propias de cada fuente (geoforma en que se presenta, accesibilidad, cantidad y diversidad de la materia prima, calidad para la talla, modo en que se presenta el recurso, entre otras) como de cuestiones dependientes del grupo que las explote (tecnología del grupo, función asignada al recurso, circuitos de movilidad, potencial simbólico del recurso, entre otras).

En cuanto a los instrumentos hallados en estratigrafía, estos deberían ser en su mayoría producidos con materia prima local y en general no deberían presentar un alto grado de formatización, mientras que los núcleos exhibirían diferentes estadios de reducción. Sin embargo, podrían hallarse instrumentos muy formatizados y con una alta inversión de energía en su manufactura en relación a funciones especificas.

Por otro lado, en áreas en que los sitios habitacionales, campamentos base o sitios de actividades múltiples se encuentran estrechamente relacionados con las canteras circundantes, las materias primas provenientes de estas se utilizarían como recursos diarios. Este sería el caso del Cañadón de la Mina, donde se encuentra CDM1. Este cañadón es concebido como un espacio total integrando concentraciones de recursos como agua, fauna, minerales, vegetales, reparo, visión. Es decir, que los antiguos habitantes de esta zona habrían acudido a la cantera para aprovisionarse de materias primas y habrían llevado éstas al sitio. En consecuencia, la expectativa es que se puedan correlacionar los conjuntos líticos de la cantera y de CDM1. Así, se esperaría observar las primeras etapas de reducción lítica en la cantera y las últimas en el sitio.

\section{LA CANTERA}

La Cantera de Sílex está localizada en el Sector Cañadón de la Mina entre el sitio CDM 1 y el bajo de la llamada Laguna Grande de La María. Su longitud máxima es de $1 \mathrm{Km}$, la zona de mayor concentración de material se encuentra a $620 \mathrm{~m}$ de CDM1. Dentro de este sector también se ubica la cantera El Morro, cerro constituido en su mayor parte por toba silicificada ubicado a $800 \mathrm{~m}$ de la cantera de Sílex y a 1400 m del sitio CDM1 (ver Fig. 2).

La Cantera de Sílex (ver Fig. 3) es una cantera primaria (Nami 1992) compuesta por abundante material suelto de color predominantemente rojo y amarillo producto del precipitado de un fluido (frío) sobresaturado en sílice (López 2004). Los nódulos más abundantes presentan un diámetro menor a $20 \mathrm{~cm}$. La calidad de la materia prima es por lo general muy buena, aunque varía dependiendo del grado de silicificación y de la homogeneidad de los nódulos (Nami 1992). La corteza que recubre a los nódulos varía en grosor y calidad para la talla, presentándose material con corteza de poco espesor y de grano fino, como el sílex rojo y material con corteza de gran espesor y grano grueso como el sílex amarillo y las tobas.

La forma en que se presentan los materiales, carente de estratigrafía (Paunero et al. 2004), así como la falta de conservación de materiales orgá-

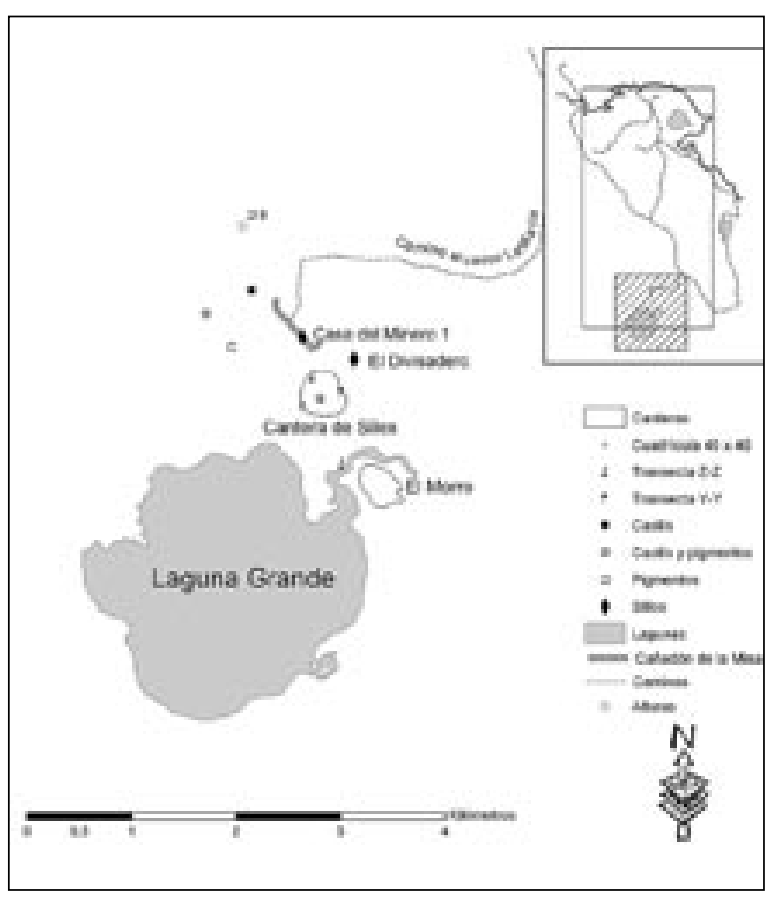

Fig. 2. Mapa del Sector Cañadón de la Mina. 

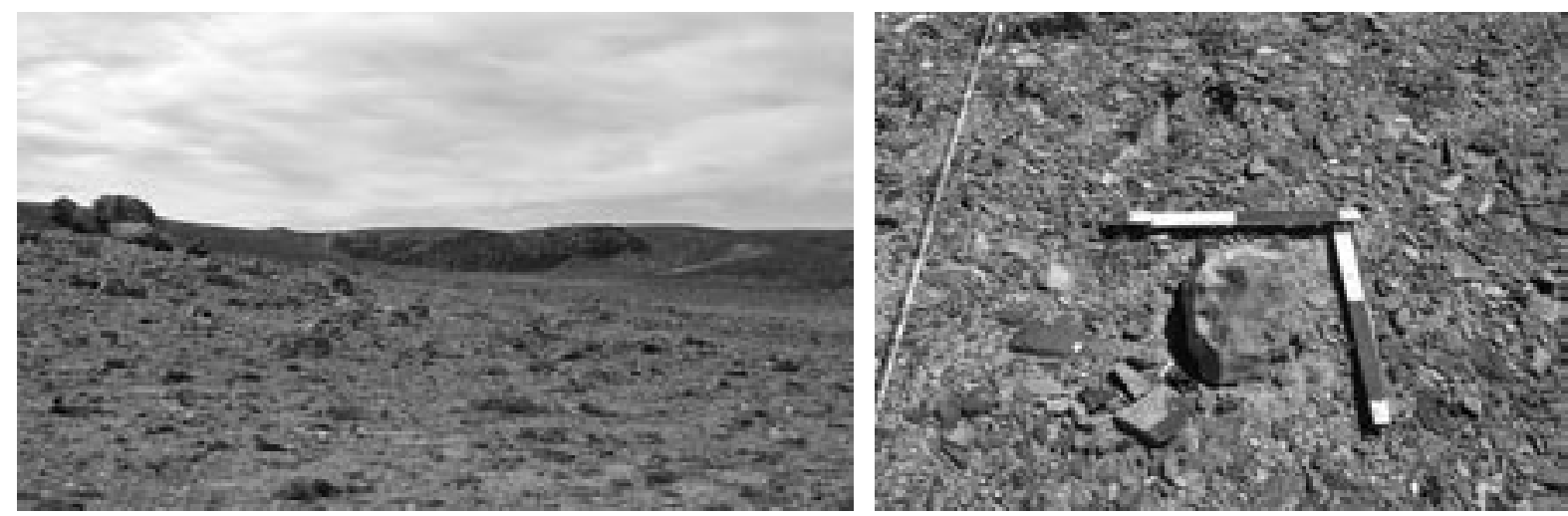

Fig. 3. Fotos de la Cantera de Sílex. a. Vista panorámica, de fondo se puede observar el Cañadón de la Mina. b. Detalle de la materia prima.

nicos potencialmente datables, impide asignar una cronología precisa a su explotación. Sin embargo, consideramos que esta cantera fue, muy probablemente, utilizada en repetidas ocasiones a lo largo del tiempo, ya que estuvo accesible en el paisaje desde las primeras ocupaciones humanas en la región y se trata de una materia prima de muy buena calidad para la talla.

Para la clasificación de los materiales se delimitó un sector de $1600 \mathrm{~m}^{2}$ en la zona central de la cantera (zona de mayor densidad), sobre la transecta principal Z - Z' que une al sitio CDM1 con la laguna (Fig. 2). En este sector se planteó una grilla subdividida en 400 cuadriculas de $4 \mathrm{~m}^{2}$, de las cuales fueron seleccionadas 113 siguiendo un muestreo sistemático. Además se clasificó el material de 21 cuadrículas del mismo tamaño realizadas cada 50 $\mathrm{m}$ a lo largo de la transecta. En cada una de ellas, se registró la totalidad de instrumentos, núcleos, productos de talla y nódulos.

El estudio de los materiales se realizó in situ, esto se debe principalmente a que consideramos que la cantera, como sitio arqueológico, constituye una parte valiosa del paisaje natural y cultural que es importante conservar, pues da una noción de su temporalidad (Ingold 2000), de las diversas culturas que habitaron el territorio, y es parte del patrimonio cultural de la región. Además estos espacios son de gran importancia pedagógica para la enseñanza de las diversas actividades llevadas a cabo en el pasado. Es por eso que consideramos conveniente levantar los materiales cuando sólo es posible estudiarlos a partir de su análisis en laboratorio.

\section{ANÁLISIS DEL CONJUNTO}

La clasificación tipológica se realizó en base a los criterios construidos en la investigación arqueológica de Patagonia (Aschero, 1975; Cardich et al. 1981-1982, Cardich y Flegenheimer 1978; Orquera y Piana 1986; Paunero y Castro 2001; Paunero et al. 2004, Skarbun et al. 2006), aunque orientados a los objetivos específicos de esta investigación.

La estructura del conjunto muestra que de un total de 2171 piezas, hay una preponderancia de los productos de talla ${ }^{1}$ (XT) con un $67,11 \%$ $(\mathrm{n}=1457)$, luego le siguen los núcleos $(\mathrm{N})$ con un $23,31 \%(n=506)$ los nódulos (No) con un $6,68 \%$ $(\mathrm{n}=145)$ y por último los instrumentos (I) con un $2,90 \%(n=63)$.

La mayor parte de los núcleos (cerca del 98\%) son poliédricos. De estos, sólo un 3\% aproximadamente está confeccionado sobre lascas nodulares, lo cual es previsible debido a las características geológicas de la cantera (el 94\% de los nódulos tiene un diámetro menor a $20 \mathrm{~cm}$ ). También se ha reconocido una muy baja cantidad de núcleos bifaciales, globulosos, prismáticos y piramidales.

En cuanto a la clase de los productos de talla, el porcentaje de lascas es muy alto, alrededor del

1 Se considera a los productos de talla como todo artefacto lítico generado como consecuencia del proceso de reducción de núcleos y de la manufactura y mantenimiento de los instrumentos. Se distinguen tres estadios: 1. estadio de descortezamiento (productos de talla con más del 90\% de corteza), 2. estadio de talla (producto de la talla del núcleo una vez descortezado), 3. estadio de retalla (productos de talla generados a partir de la formatización final, reactivación y mantenimiento de los instrumentos). 


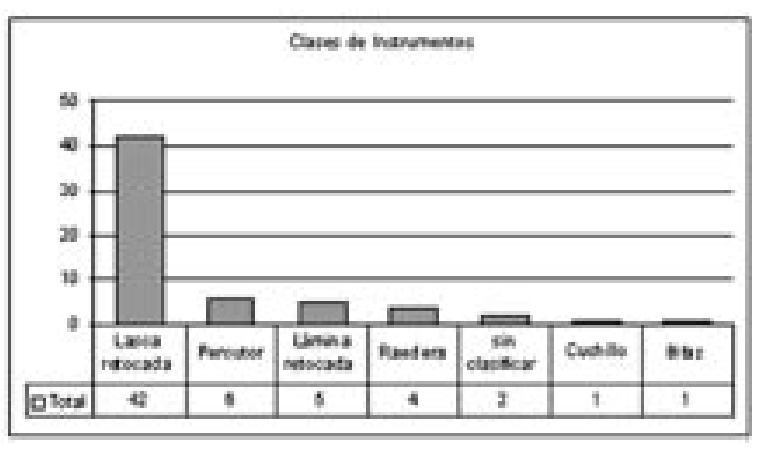

Fig. 4. Cantidad de Instrumentos por clase en la cantera.

90\% y hay aproximadamente un 5\% de láminas. El resto (lascas largas, anchas, triangulares, microlascas y laminillas) no superan cada una el $2 \%$.

Predominan los instrumentos poco formatizados (lascas y láminas retocadas), aunque se reconocen escasos instrumentos con mayor inversión energética como raederas, cuchillos y bifaces; cabe señalar la presencia de seis percutores (Fig. 4).

Las materias primas fueron clasificadas y determinadas macroscópicamente según el tipo de roca (sílex, toba, toba silicificada y otras) y se diferenciaron los colores más representados para el sílex (que es el tipo de roca más abundante). En el caso en que la pieza presentara más de un color se la clasificó como variedad del color principal.

El sílex está presente en el 75\% de la muestra. En la Tabla 1 se puede observar que los más abundantes son los sílex rojos y los sílex amarillos (cada uno con un 25\% aproximadamente, considerando sus variedades). El sílex marrón con su variedad alcanza un 10\% aproximadamente. Las distintas variedades de tobas y tobas silicificadas no llegan al 15\%. Los demás tipos de materias primas en su conjunto no superan el 5\%.

El análisis de la Tabla 1, muestra también, que la materia prima predominante en los instrumentos, los núcleos y los nódulos es el sílex rojo. En cambio, en los productos de talla predomina el sílex amarillo. Por otra parte, se observa que casi el $10 \%$ de los instrumentos corresponden a la categoría de otras materias primas. En este sentido, hay que tener en cuenta que aquí se consideran los percutores que no corresponden a materias primas de esta cantera.

Se clasificaron los núcleos según el estado de sus plataformas de reducción. Se utilizaron tres estadios: 1: Reducción Inicial, cuando las plataformas de reducción son en su mayoría corticales; 2: Núcleos Activos, cuando la mayoría de sus plataformas están activas, pudiendo permanecer una minoría corticales y 3: Núcleos Agotados, cuando no hay plataformas adecuadas para la percusión. La mayor parte de los núcleos (51,29\%) corresponde al estadio 2, el $25,15 \%$ corresponde al estadio 1 y el 15,84\% están agotados. El 7,72\% son indeterminados.

En relación a los productos de talla, la mayoría (ver Tabla 2) corresponde al estadio 2 de talla (más del 50\%), un 37\% corresponde al estadio 1 de descortezamiento y apenas un $2 \%$ al estadio 3 de formatización final. Además, casi el 15\% de las piezas correspondientes al estadio de talla presenta corteza. También cabe destacar que hay un alto porcentaje de talones lisos (42\%) y naturales (36\%) que son característicos de las primeras etapas de reducción.

Tabla 1. Porcentajes de materias primas dentro de los grupos.

\begin{tabular}{|l|c|c|c|c|}
\hline \multicolumn{1}{|c|}{ Materia Prima } & $\mathrm{I}$ & $\mathrm{N}$ & No & XT \\
\hline Sílex amarillo & $18,03 \%$ & $17,39 \%$ & $9,66 \%$ & $23,65 \%$ \\
\hline Sílex amarillo variedad & $1,64 \%$ & $3,36 \%$ & $2,07 \%$ & $2,54 \%$ \\
\hline Sílex rojo & $27,87 \%$ & $32,61 \%$ & $51,72 \%$ & $18,44 \%$ \\
\hline Sílex rojo variedad & $1,64 \%$ & $2,77 \%$ & $2,76 \%$ & $1,78 \%$ \\
\hline Sílex marrón & $13,11 \%$ & $4,74 \%$ & $2,07 \%$ & $8,29 \%$ \\
\hline Sílex marrón variedad & $4,92 \%$ & $3,56 \%$ & $4,83 \%$ & $5,21 \%$ \\
\hline Otras materias primas & $9,84 \%$ & $0,59 \%$ & $2,07 \%$ & $0,41 \%$ \\
\hline Toba silicificada & $4,92 \%$ & $16,01 \%$ & $6,90 \%$ & $14,05 \%$ \\
\hline Otros Sílex & $14,75 \%$ & $12,45 \%$ & $11,72 \%$ & $15,22 \%$ \\
\hline Toba & $1,64 \%$ & $6,13 \%$ & $4,83 \%$ & $9,94 \%$ \\
\hline Indeterminadas & $1,64 \%$ & $0,40 \%$ & $1,38 \%$ & $0,48 \%$ \\
\hline Total & $100,00 \%$ & $100,00 \%$ & $100,00 \%$ & $100,00 \%$ \\
\hline
\end{tabular}


Tabla 2. Estadios de reducción de los productos de talla con clase determinada según las materias primas.

\begin{tabular}{|l|c|c|c|c|c|c|c|c|c|c|}
\hline \multicolumn{1}{|c|}{ Materia Prima } & \multicolumn{2}{|c|}{1} & \multicolumn{2}{c|}{2} & \multicolumn{2}{c|}{3} & \multicolumn{2}{c|}{ Sin clasificar } & \multicolumn{2}{c|}{ Total } \\
\hline & Cant. & $\%$ & Cant. & $\%$ & Cant. & $\%$ & Cant. & $\%$ & Cant. & $\%$ \\
\hline Sílex amarillo & 112 & 43,08 & 137 & 52,69 & 4 & 1,54 & 7 & 2,69 & 260 & 100 \\
\hline Sílex amarillo variedad & 15 & 50,00 & 13 & 43,33 & 1 & 3,33 & 1 & 3,33 & 30 & 100 \\
\hline Sílex rojo & 60 & 28,44 & 139 & 65,88 & 6 & 2,84 & 6 & 2,84 & 211 & 100 \\
\hline Sílex rojo variedad & 9 & 34,62 & 16 & 61,54 & - & 0,00 & 1 & 3,85 & 26 & 100 \\
\hline Sílex marrón & 27 & 28,42 & 64 & 67,37 & 2 & 2,11 & 2 & 2,11 & 95 & 100 \\
\hline Sílex marrón variedad & 23 & 34,33 & 39 & 58,21 & 1 & 1,49 & 4 & 5,97 & 67 & 100 \\
\hline Toba silicificada & 39 & 23,93 & 118 & 72,39 & 1 & 0,61 & 5 & 3,07 & 163 & 100 \\
\hline Otros Sílex & 51 & 32,28 & 101 & 63,92 & 3 & 1,90 & 3 & 1,90 & 158 & 100 \\
\hline Toba & 85 & 67,46 & 39 & 30,95 & - & 0,00 & 2 & 1,59 & 126 & 100 \\
\hline Total & 421 & 37,06 & 666 & 58,63 & 18 & 1,58 & 31 & 2,73 & 1136 & 100 \\
\hline
\end{tabular}

En general las materias primas tienen mayores porcentajes en los estadios de talla. Sin embargo, esto no ocurre con el sílex amarillo variedad y las tobas, que tienen mayores porcentajes de lascas de descortezamiento; seguramente debido a que estos materiales presentan, como ya se ha mencionado, una corteza muy espesa que era necesario eliminar. Esto explica también porqué hay más cantidad de productos de talla de sílex amarillo que de sílex rojo. Si se observa la Tabla 2 puede verse que la cantidad de productos de talla de los estadios 2 y 3 es prácticamente la misma para ambas materias primas, mientras que en el estadio de descortezamiento la cantidad de productos de talla de sílex amarillo es casi el doble que la de sílex rojo.

\section{COMPARACIÓN CON EL SITIO CASA DEL MINERO 1}

El sitio CDM1 es un sitio multicomponente que presenta ocupaciones que van desde el Pleistoceno Final hasta momentos históricos (Paunero 2000, 2003, Paunero et al. 2001, 2004; Paunero et al. 2005; Skarbun et al. 2006). El conjunto lítico del sitio se caracteriza por presentar en general un alto porcentaje de productos de talla correspondiente al estadio de formatización final, así como del estadio de talla, mientras que las evidencias de descortezamiento son muy escasas. Los instrumentos hallados en el sitio son principalmente lascas retocadas, raederas y raspadores (Ver Fig. 5). Sólo se encontró un núcleo, lo que dificulta la comparación de éstos con los de la cantera.

Teniendo en cuenta que CDM1 y la cantera de Sílex se encuentran en la misma unidad paisajística, se realizó un análisis macroscópico que permitió observar una gran similitud en las materias primas de ambos sitios. Por lo tanto, planteamos a manera de hipótesis que la mayor parte de los materiales líticos recuperados del sitio CDM1 provienen de esta cantera. En consecuencia, la expectativa es que se puedan correlacionar ambos conjuntos líticos, esperándose observar las primeras etapas de reducción lítica en la cantera y las últimas en Casa del Minero 1. En esta sección realizamos un análisis comparativo de los materiales registrados en estos sitios para aportar datos que permitan corroborar o refutar esta hipótesis.

Por otro lado, cabe aclarar que en relación al establecimiento de la procedencia de las materias primas de esta localidad (grupo Bahía Laura), no se han realizado por el momento estudios detalla-

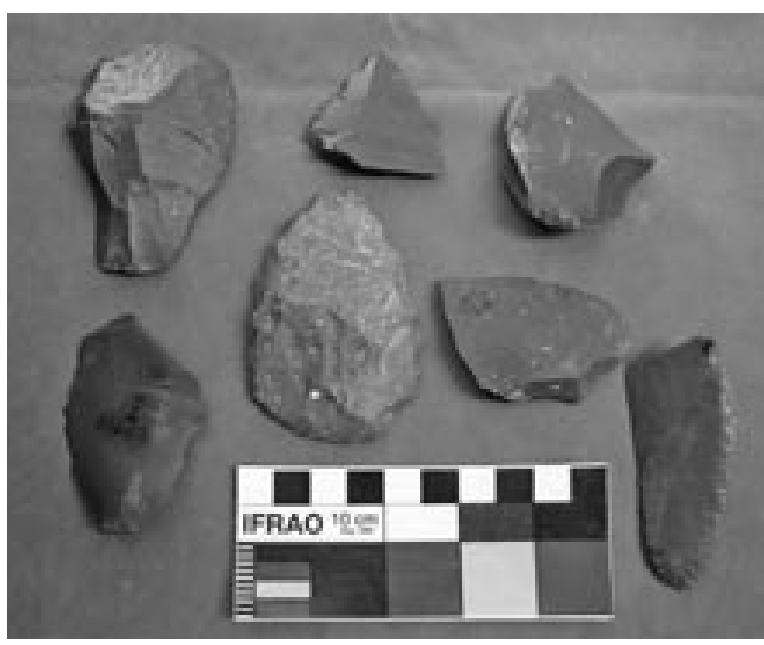

Fig. 5. Foto de instrumentos de sílex rojo provenientes de Casa del Minero 1. 


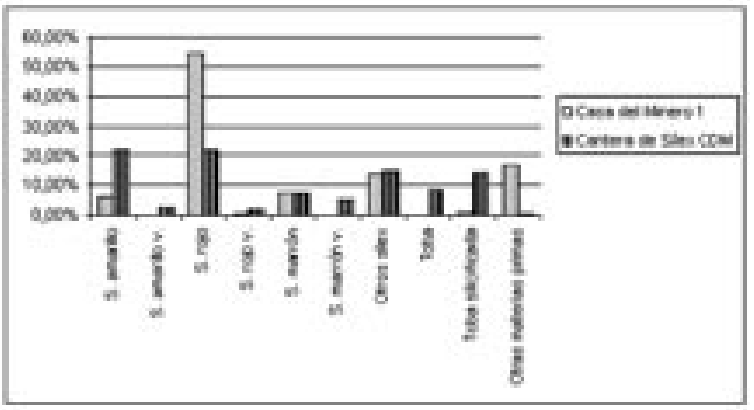

Fig. 6. Comparación de materias primas entre Casa del Minero 1 y Cantera de Sílex.

dos (como la utilización de cortes petrográficos) o analíticos (como la geoquímica de elementos trazas y mayoritarios), aunque futuros análisis están contemplados.

Si bien existen diferencias entre los conjuntos de los niveles 2, 3 y 4 de CDM1 (Paunero et al. 2001, 2004, Skarbun et al. 2006), éstos comparten similitudes en las variables (materias primas, estadios de reducción, grupos y clases tipológicas) utilizadas para realizar la comparación entre el sitio y la cantera, por lo que es posible agrupar todos los conjuntos.

Al comparar los porcentajes de las materias primas entre estos sitios (Fig. 6) se observa que CDM1 tiene mayor porcentaje de sílex rojo que la cantera, mientras que el porcentaje del sílex amarillo se comporta de manera inversa. Ambos rasgos son llamativos si se tiene en cuenta que en la actualidad, en la cantera hay aproximadamente la misma cantidad de materiales de estos sílex. Así, parecería factible que se haya seleccionado principalmente el sílex rojo para su utilización. Para el resto de los sílex el porcentaje es similar en ambos lados. Por otra parte tanto la toba como la toba silicificada tienen porcentajes considerables en la cantera mientras que son casi inexistentes en el sitio.

En cuanto a los estadios de reducción de los productos de talla presentes en ambos lados, los porcentajes concuerdan con lo esperado: hay abundancia de productos de talla del estadio 1 en la cantera y del estadio 3 en CDM1 (ver Fig. 7). Los porcentajes del estadio 2 son similares en ambos casos, si bien, cuando se compara el tamaño de los productos de talla de este estadio, se vuelve visible que estos son más grandes en la cantera que en el sitio. Esto es razonable, puesto que los restos

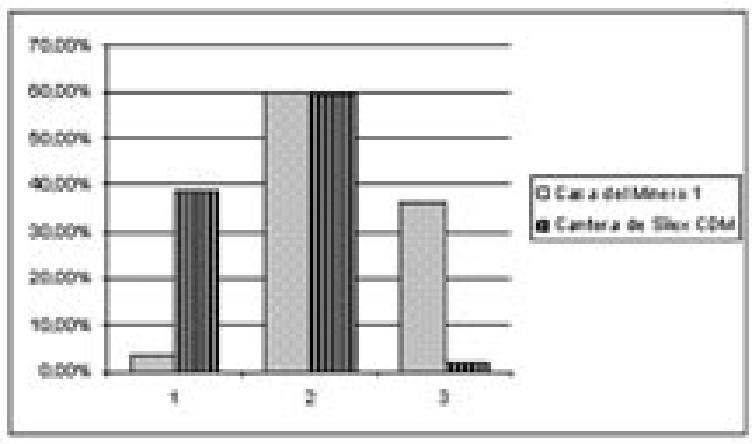

Fig. 7. Comparación de los estadios de reducción de los productos de talla en CDM 1 y la cantera de Sílex.

del estadio 2 de la cantera están más relacionados con la reducción inicial mientras que los del sitio se vinculan más a la formatización final.

\section{DISCUSIÓN}

La abundancia de núcleos y de lascas primarias, indican que la principal actividad llevada a cabo en la cantera fue el aprovisionamiento de nódulos para la talla así como las primeras etapas de reducción. También respalda esta afirmación el alto porcentaje de talones naturales y lisos (característicos de las primeras etapas de reducción) que se reconocieron entre los productos de talla y la presencia de corteza en las piezas del estadio de talla.

Cabe destacar que se han identificado gran cantidad de núcleos que presentan un solo negativo de lascado, lo que demuestra que se testeaban los nódulos antes de continuar con su reducción. Esto se observa especialmente en aquellos núcleos que por fuera presentan una corteza de toba de muy mala calidad pero que rodea un interior de sílex (mayormente amarillo) que es apto para la talla. Este hecho explica también porqué se encuentran proporcionalmente en el estadio de reducción inicial más núcleos de sílex amarillo que de sílex rojo, porqué tantos productos de talla de toba corresponden al estadio de descortezamiento así como la preponderancia de productos de talla de sílex amarillo por sobre los de sílex rojo. Es decir, esta materia prima demandaba una mayor inversión de tiempo y energía para su descortezamiento.

Por otro lado, el hecho de que la mayor parte de los núcleos hallados sean poliédricos y no estén agotados indicaría que han sido usados 
expeditivamente; además, estos núcleos quedarían disponibles para su utilización en otros momentos. Esto es lo que se espera en los casos en que existe alta disponibilidad de materias primas de buena calidad en la zona.

Además, en general no se han encontrado productos de talla muy regularizados (bajo porcentaje de laminaridad y solo dos lascas triangulares), lo cual también indicaría que se le dio un uso más expeditivo a la cantera.

Queda la incógnita acerca de la reducción de ciertos núcleos extremadamente pequeños. Una posibilidad es que estos núcleos hubiesen sido preferidos por tener alguna cualidad diferencial. Otra posibilidad es que hayan sido utilizados para la confección de instrumentos muy pequeños (microlitos) aunque hasta el momento éstos no han sido hallados en el registro arqueológico de la localidad.

Si bien la cantidad de percutores reconocidos es escasa, hay que tener en cuenta dos factores: por un lado los percutores formarían parte del equipo personal que el tallador habitualmente lleva consigo, y en consecuencia no sería común dejarlos en la cantera; y por el otro, los percutores son nódulos de materias primas que hasta hoy no han sido reconocidas en el sector, o sea, es posible que sean alóctonas. Por lo tanto, siendo transportados, es probable que estos no hayan sido descartados con frecuencia. Por otra parte, el número de percutores aumentaría si aquellos nódulos que se clasificaron como Potenciales Percutores Funcionales ${ }^{2}$ (PPF) resultaran ser efectivamente este tipo de instrumento. Si ese fuese el caso la afirmación precedente sufre un revés puesto que no presentan marcas, no están agotados y en general no se encuentran fracturados, por lo que su abandono no estaría justificado, y podríamos considerar como factible una hipótesis de equipamiento del espacio para el caso de los percutores.

También se han reconocido instrumentos, por lo que se infiere que se han realizado otras actividades en la cantera. Teniendo en cuenta que en su mayoría son lascas retocadas que demandan un bajo costo en su producción, y también considerando la cercanía de la laguna, es factible que se hayan realizado otras actividades en los alrededores.

2 Nódulos hallados en la cantera, de materia prima alóctona y morfológicamente factibles de ser percutores pero que no presentan marcas que permitan identificarlos como tales.
Además, se reconocieron abundantes productos de talla con rastros en los bordes. Debido a que se trata de una clasificación realizada in situ dada por la orientación de este trabajo, no se pudo determinar si estos rastros son producto del uso o si son consecuencia de procesos postdepositacionales. Sin embargo, es probable que al menos algunos de ellos se hayan producido durante la utilización de filos naturales. En consecuencia, se podría decir que en ciertos casos el aprovisionamiento de la materia prima estuvo incorporado a otras actividades como el procesamiento primario de presas.

Teniendo en cuenta la disponibilidad y accesibilidad de la materia prima (y cercanía si se considera CDM1), el aprovisionamiento exigiría poco esfuerzo, es decir que sería de bajo costo energético. Consecuentemente, no se consideran pertinentes para este caso las discusiones concernientes a las estrategias implementadas para el aprovisionamiento de materias primas (por ejemplo viajes incorporados versus específicos) (Binford 1979; Morrow y Jefferies 1989).

Por otro lado en la cantera se han encontrado materiales provenientes de El Morro, lo cual implicaría el uso de esta como taller lítico, habiéndose trasladado material hacia la Cantera de Sílex donde se habría tallado.

Teniendo en cuenta que en esta cantera se realizaron principalmente tareas de aprovisionamiento y de reducción inicial, es esperable que la mayor parte de los instrumentos se encuentren en los campamentos base, sitios de actividades múltiples o específicas. A partir de los análisis realizados en CDM1 y considerando que éste se encuentra en la misma unidad paisajística de la cantera, mantenemos la hipótesis acerca de que la mayor parte de los artefactos del sitio fueron confeccionados con materia prima probablemente proveniente de ésta. Sin embargo, habría existido selectividad en el transporte de materias primas desde la cantera hacia el sitio, ya que algunas se encuentran en ella y no en CDM1 (toba, toba silicificada) o bien se encuentran en menor proporción (sílex amarillo), mientras que algunas materias primas tienen una importancia relativamente mayor en el sitio que en la cantera (sílex rojo).

Esto podría indicar que la toba y la toba silicificada fueron manipuladas principalmente en la cantera. En el caso de la toba silicificada se habría 
usado circunstancialmente allí a pesar de su menor calidad para la talla con relación al sílex; en el caso de la toba quizás los restos hallados se generen exclusivamente como consecuencia del descortezamiento de nódulos en busca de las partes de mejor calidad de la materia prima, otra posibilidad podría ser que en distintos momentos a lo largo del holoceno se diera un uso diferente a estas rocas. Las "otras materias primas" están más representadas en CDM1, lo cual es esperable ya que en la cantera se aprovechan recursos in situ mientras que el sitio sería parte de un circuito más amplio en el cual recibiría materias primas de otras fuentes alejadas.

Estos datos permitirían afirmar que la cantera de sílex jugó un papel importante en la configuración del conjunto lítico de CDM1 (Skarbun et al. 2006). La escasez de núcleos y la abundancia de restos pertenecientes a los estadios finales de formatización en este sitio hacen suponer que desde la cantera se trasladaron principalmente formas base y que allí se terminó de dar forma a los instrumentos.

Si bien los instrumentos hallados en CDM1 podrían enmarcarse en mayor medida dentro de estrategias expeditivas (Skarbun et al. 2006), el hecho que se hallen productos de talla de adelgazamiento bifacial, instrumentos bifaciales y otros con alta inversión de energía en su producción (como por ejemplo cuchillos, raspadores-raederas y puntas de proyectil), lleva a pensar en la posibilidad de una estrategia combinada. Así, ciertos instrumentos tendrían mayor conservación, dependiendo de la función a la que estaban destinados. Estas estrategias deberán ser analizadas en relación al lugar que ocupó CDM1 dentro del circuito de movilidad regional.

\section{CONCLUSIÓN}

Los datos generados en este trabajo permiten concordar con lo propuesto previamente acerca de la funcionalidad de la Cantera de Sílex. Esta ha servido como sitio de extracción y como taller lítico de los estadios iniciales en los procesos de elaboración de instrumentos desde las primeras ocupaciones. $\mathrm{Su}$ explotación fue expeditiva, lo cual se evidencia a partir de las características tecnológicas de los restos líticos encontrados allí. El hallazgo de instrumentos en la cantera también indicaría que se realizaron otros tipos de actividades, las cuales podrían estar relacionadas con el procesamiento primario de presas cazadas. Además con los datos actualmente disponibles y a partir del análisis realizado, se mantiene la hipótesis que la materia prima proveniente de esta cantera fue intensamente utilizada en los procesos de producción lítica de las ocupaciones del sitio CDM1. Futuros estudios geológicos y geoquímicos de las materias primas de la cantera y de CDM1 nos permitirán aportar mayor confiabilidad a esta hipótesis.

Los estudios de canteras resultan útiles para analizar las primeras etapas de la organización de la tecnología lítica. Además, este tipo de estudios permite analizar la vinculación entre éstas y otros sitios, aportando entonces al análisis de las distintas estrategias de movilidad, subsistencia y uso del espacio que practicaron los grupos que habitaron una región. Más específicamente, el análisis realizado en este trabajo se cree que ha contribuido a una mejor comprensión de las estrategias tecnológicas adoptadas en lugares de alta disponibilidad de materias primas de muy buena calidad.

\section{AGRADECIMIENTOS}

Agradecemos a todo nuestro equipo de investigación por el apoyo brindado y por las arduas jornadas de clasificación de material en la cantera; al Dr. Ramiro López por las observaciones geológicas; a la Dra. Alicia Castro por la lectura y corrección del trabajo; al Lic. Rafael Paunero por apoyarnos y guiarnos en la investigación y finalmente a la Familia Behm por el apoyo brindado a nuestras investigaciones y su amable disposición al recibirnos en su estancia La María.

\section{BIBLIOGRAFÍA}

ANDREFSKY, W. 1991. Inferring trends in prehistoric settlement behavior from lithic production technology in the southern plains. North American Archaeology 12 (2): $129-144$

ANDREFSKY, W. JR. 1994. The Geological Occurrence of Lithic and Stone Tool Production Strategies. Geoarchaelogy: An International Journal 9 (5): 375-391. Editado por John Wiley and Sons, Inc.

ASCHERO, C. 1975. Ensayo para una clasificación morfológica de artefactos líticos aplicado a estudios tipológicoscomparativos. Informe presentado al CONICET. MS. Buenos Aires. 
BAMFORTH, D. B. 1986. Technological efficiency and tool curation. American Antiquity 51 (1): 38-50.

BELARDI, J. B. y CARBALLO MARINA, F. 2005. Canteras Taller de Basalto en la zona de Bajo Caracoles - Río Olnie (Provincia de Santa Cruz). Intersecciones en Antropología 6: 223-226. Facultad de Ciencias Sociales, UNCPBA, Argentina.

BINFORD, L. R. 1977. Forty-Seven trips. Stone tools as Cultural Markers. R. V. S. Wright (Ed.), pp. 24-36. Australian institute of Aboriginal Studies. Canberra, Australia.

BINFORD, L. R. 1979. Organization and Formation Processes: Looking at Curated Technologies. Journal of Anthropological Research 35 (3): 255- 273

BINFORD, L. R. 1980. Willow Smoke and Dog's Tails: HunterGatherer Settlement Systems and Archaeological Site Formation. American Antiquity 45 (1): 4-20.

BOUSMAN, C. B. 1993. Hunter-Gatherer Adaptations, Economic Risk and Tool Design. Lithic Technology 18 (1 y 2): 59-86.

CARDICH, A. y FLEGENHEIMER, N. 1978. Descripción y tipología de las industrias más antiguas de Los Toldos. Relaciones de la S.A.A. XII: 225-242.

CARDICH, A.; MANSUR-FRANCHOMME, M.; DURÁN, V. y GIESSO, M. 1981-82. Arqueología de las cuevas de El Ceibo, Santa Cruz, Argentina. Relaciones de la S.A.A. XIV (2): 241-267. Bs. As.

CARDICH, A. y PAUNERO, R. 2000. Capítulo 6.1: Localidad Arqueológica La María. Introducción. Guía de Campo de la Visita a las Localidades Arqueológicas: La Colonización del Sur de América Durante la Transición Pleistoceno/Holoceno. L. Miotti, R. Paunero, M. Salemme y R. Cattáneo (Eds.). La Plata.

CATTANEO, R. 2004. Desarrollo metodológico para el estudio de fuentes de aprovisionamiento lítico en la meseta central santacruceña, Patagonia argentina. Estudios Atacameños 28: 105-119.

ESPINOSA, S., J. B. BELARDI y F. CARBALLO MARINA. 2001. Fuentes de aprovisionamiento de materias primas líticas en los sectores medio e inferior del interfluvio Coyle-Gallegos (departamento Güer Aike, provincia de Santa Cruz). Desde el País de los Gigantes. Perspectivas arqueológicas en Patagonia. J. B. Belardi, F. Carballo Marina y S. Espinosa (Eds.), pp. 5-17. Editorial Universidad Nacional de la Patagonia Austral. Río Gallegos.

ESPINOSA, S. y R. GOÑI. 1999. ¡Viven!: Una fuente de obsidiana en la provincia de Santa Cruz. Soplando en el viento...Actas de las Terceras Jornadas de Arqueología de la Patagonia. Pp 177-188.
FRANCO, N. y E. ARAGON. 2004. Variabilidad en fuentes secundarias de aprovisionamiento lítico: El caso del sur del lago Argentino (Santa Cruz, Argentina). Estudios Atacameños 28: 71-85.

HERMO, D. 2005. Acerca del aprovechamiento de xilópalo en el Monumento Natural Bosque Petrificado, por parte de los cazadores-recolectores de la meseta central de Santa Cruz. Trabajos de las VI Jornadas de Jóvenes Investigadores en Ciencias Antropológicas.

INGOLD, T. 2000. The temporality of landscape. Interpretive archaeology: a reader. Julian Thomas (Ed.), pp 510-530. Leicester University Press. London and New York.

KELLY, R. L. 1988. The three sides of a biface. American antiquity 53 (4): 717-735.

LÓPEZ, R. 2004. Informe Sobre Afloramientos Rocosos de Interés Arqueológico en Estancia La María, Santa Cruz. M.S.

MORROW, C. y R. JEFFERIES. 1989. Trade or embedded procurement?: a test case from southern Illinois. Time, Energy and Stone tools. Robin Torrence (Ed.). Cambridge University Press. Cambridge.

NAMI, H. 1992. El subsistema tecnológico de la confección de instrumentos líticos y la explotación de los recursos del ambiente: una nueva vía de aproximación. Shincal 2: $13-53$

NELSON, M, 1991. The study of technological organization. Archaeological Method and Theory 3:57-100. Ed. M. Schiffer. University of Arizona Press. Tucson.

ORQUERA, L. y PIANA, E. 1986. Normas para la descripción de objetos arqueológicos de piedra tallada. CADIC. Contribución Científica № 1 (Publicación Especial), Ushuaia.

PAUNERO, R. 2000. Capítulo 6.5: Cañadón de La Mina: Sitio Casa del Minero 1. Guía de Campo de la Visita a las Localidades Arqueológicas: La Colonización del Sur de América Durante la Transición Pleistoceno/Holoceno. L. Miotti, R. Paunero, M. Salemme y R. Cattáneo (Eds.). La Plata.

PAUNERO, R. S. y CASTRO, A. 2001. Análisis lítico y funcionalidad del componente inferior de Sitio Cueva 1, Localidad Arqueológica Cerro Tres Tetas, Provincia de Santa Cruz, Argentina. Anales del Instituto de la Patagonia. Serie Ciencias Humanas 29: 189-206. Punta Arenas. Chile.

PAUNERO, R., ALBERTENGO, P., CUETO, M., DÁVILA, A., FRANK, A., OLIVERA, A. y PIVA C. 2001. Sitio Casa del Minero 1, localidad arqueológica La María: nuevas evidencias sobre ocupación humana pleistocénica en Santa Cruz. XIV Congreso Nacional de Arqueología 
Argentina. Facultad de Humanidades y Artes. Universidad Nacional de Rosario. Rosario.

PAUNERO, R.; CUETO, M.; FRANK, A.; SKARBUN, F.; GHIDINI, G.; y ROSALES, G. 2004. Comunicación sobre campaña arqueológica 2002 en localidad La María, Santa Cruz. Contra viento y marea. Arqueología de Patagonia. Pp. 797-808 Instituto Nacional de Antropología y Pensamiento Latinoamericano. Buenos Aires.

PAUNERO, R. S., FRANK, A. D., SKARBUN, F., ROSALES, G., ZAPATA, G., CUETO, M., PAUNERO, M. F., MARTÍNEZ, D. G., LÓPEZ, R., LUNAZZI, N. y DEL GIORGIO, M. 2005. Investigaciones arqueológicas en Estancia La María, Meseta Central de Santa Cruz. Relaciones de la S.A.A. Tomo XXX: 147-168. Buenos Aires.
SKARBUN, F., FRANK, A. D. y PAUNERO M. F. 2006. Análisis de la tecnología lítica del sitio Casa del Minero 1, Meseta Central de Santa Cruz. VI Jornadas de Arqueología de la Patagonia. Punta Arenas, En Prensa.

STERN R., C. 2004. Obsidian in Southern Patagonian: Review of the Current Information. Contra viento y marea. Arqueología de Patagonia. Pp. 167-176. Instituto Nacional de Antropología y Pensamiento Latinoamericano. Buenos Aires. 